\title{
Passive and Active Controls of Three-Dimensional Wake of Bluff-Body*
}

\author{
Hiroshi HIGUCHI ${ }^{* *}$
}

\begin{abstract}
Passive and active controls of wakes behind axisymmetric bluff-bodies are presented. The passive controls include base-bleed (slotted disks), edge-induced longitudinal vortices behind three-dimensional polygonal plates, the fineness ratio and surface protrusion. The disk wake was further actively controlled in air using actuated tabs and in water using net-zero mass actuators along the edge. The active control suppressed the helical mode of oscillation, but enhanced the closing of the reverse flow region with reduced base pressure. Unsteady velocity vector fields from the time-resolved PIV are presented and issues related to future feedback flow control for the bluff-body wake will be addressed.
\end{abstract}

Key Words: Wake, Separation, Flow Control, Axisymmetric Flow, Three-Dimensional Flow

\section{Introduction}

While the wake behind two-dimensional bluff-bodies including its three-dimensional state has been extensively studied, less is known regarding the wake behind axisymmetric and three-dimensional bluff-bodies, in particular the control aspect of the wake flow. The wake behind the axisymmetric body becomes three-dimensional with multiple scales of the vortical structures ${ }^{(1),(2)}$. Higuchi and Balligand ${ }^{(3)}$ demonstrated the establishment of the 3dimensional wake behind a disk started from rest. In addition, the bluff-body wake with large reverse flow region exhibiting absolute instability (see e.g., Ref. (4)), its control poses an increased level of challenge. One method of the wake control behind the body with continuous surface is the boundary layer and separation modification. Examples of passive control of the wake include roughness and seams on sports balls. The result of the experiment on a baseball will be shown. The three-dimensional geometry or protrusion can force vortex stretching and longitudinal vortices. Another control method is based on base-bleed. For a sharp-edged disk, passive control of the disk wake has been demonstrated by Bevilaqua ${ }^{(5)}$ and Cannon ${ }^{(6)}$ by adding porosity. Here the porosity produced by a slotted disk will be presented. This type of wake control, as well as controlling the fluid-interaction problem, has been uti-

* Received 22nd November, 2004 (No. 04-5185)

** Department of Mechanical and Aerospace Engineering, Syracuse University, Syracuse, NY 13244, U.S.A.

E-mail: hhiguchi@syr.edu lized in parachute applications ${ }^{(7),(8)}$.

Active control of the boundary layer separation on the continuous surface was recently demonstrated by Jeon et al. ${ }^{(9)}$ They applied periodic suction and blowing over a sphere surface and achieved a drag reduction. For a sharp-edged bluff-body, however, the separation point is fixed and the boundary layer control becomes ineffective. Berger et al. ${ }^{(1)}$ oscillated the disk itself and the found the nutation mode to be most effective in enhancing the disk wake structure. This disk oscillation can be regarded as time-dependent control of the separation line. In the present experiment, the disk edge is equipped with an actuated edge to control the separating shear layer while the separation point is fixed. This paper reviews the various passive and active control methodologies based on the author's work and addresses future possibilities and challenges. Reynolds number in these examples was in the order of $10^{4}$ to $10^{5}$.

\section{Passive Control}

\section{1 Wake behind slotted disk (Base-bleed)}

One of the conventional methods of the bluff-body wake control is a base-bleeding. The absolutely unstable flow is rendered into a convectively unstable flow. An example of the wake behind a slotted disk is shown in Fig. 1. Here the flow is from left to right, and the hydrogen bubble visualization depicts the gap flows through slots that merge outward except for the center jet. Beside the reduced drag, the large wake oscillations downstream were suppressed. Some flip-floppings of the flow patterns were observed at the same time. Even with the same geometry 


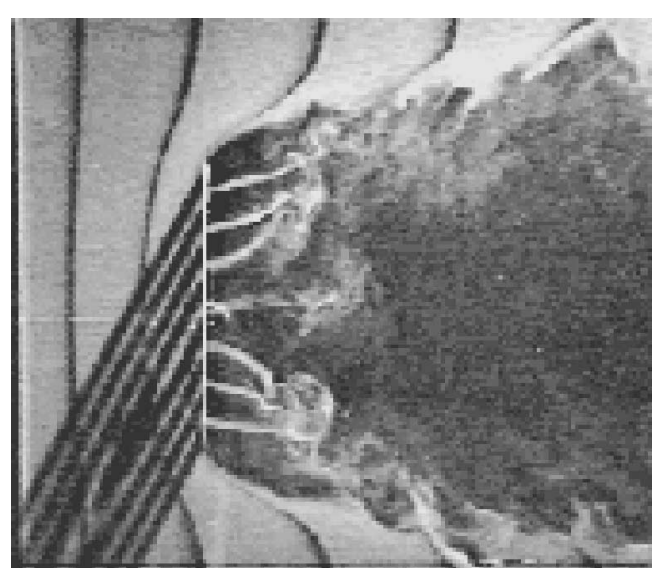

Fig. 1 Flow past a slotted disk (spacing ratio 0.5, $R e=7.4 \times 10^{3}$ )

and the free stream, the wake structure and energy spectrum depended on the specific wake pattern. More detailed discussion for this as well as for other slotted disk configuration is given in Ref. (10).

A special case of a base bleed occurs when the bleed momentum is matched with the drag, producing so-called a momentumless wake ${ }^{(11),(12)}$. In the latter, a slight excess momentum produced a co-flowing jet structure, while a wake structure resulted from a reduced momentum. In the matched momentum case, the downstream wake was devoid of production of large-eddy structure.

\subsection{Effect of length to diameter ratio}

If the disk width is increased, the geometry becomes that of a truncated cylinder.

Flow over a circular cylinder aligned with the free stream was examined using the Japan Space Exploration Agency's magnetic suspension and balance system ${ }^{(13),(14)}$. The model set up is shown in Fig. 2. While the parameters are simply the length to diameter ratio, $L / D$, and the Reynolds number, the phenomenon involves the leading edge separation and reattachment if the $L / D$ is sufficiently large, and the recirculation region in the immediate wake. Figure 3 indicates the present measurements and previously available data, which go back to Eiffel's experiment one century ago. The reattachment of the leading edge separation reduces the pressure drag, while at larger $L / D$, the wall shear stress on the cylinder surface causes the increased overall drag. The drag coefficient past 2-dimensional rectangular plate reaches maximum at the fineness ratio at approximately 0.6 . It will be also of interest to ascertain the shear layer behavior in this region.

Effect of the fineness ratio the wake behind short cylinders in supersonic free flight was also addressed in a preliminary study ${ }^{(15)}$.

\subsection{Polygonal plate and longitudinal vortices}

As stationary tabs were used to manipulate the jet spreading rate and mixing, the planform of the plate was found to greatly influence the wake development. The

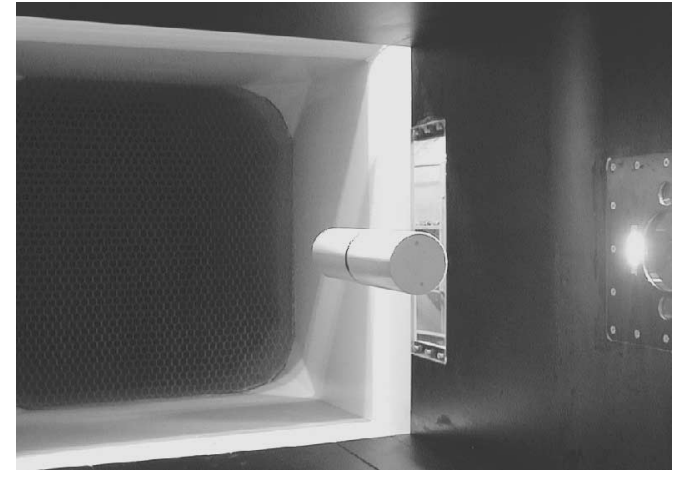

Fig. 2 A magnetically supported cylinder model

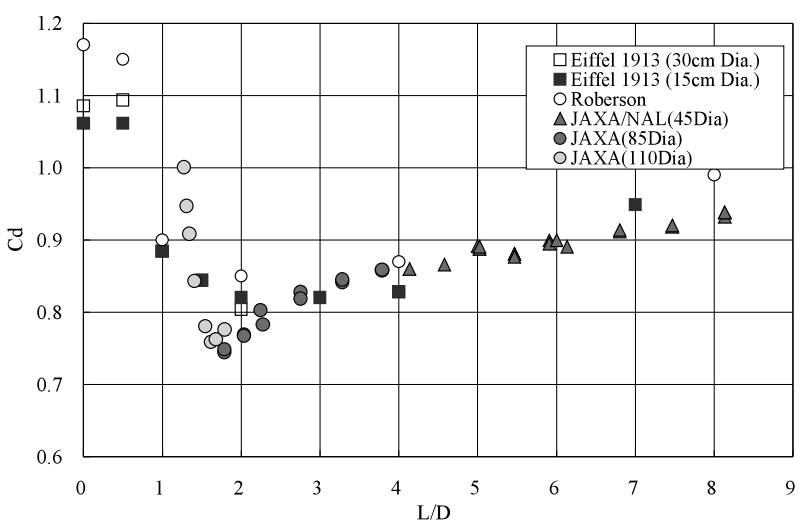

Fig. 3 Circular cylinder in axial flow: dependence of drag coefficient on fineness ${ }^{(14)}$

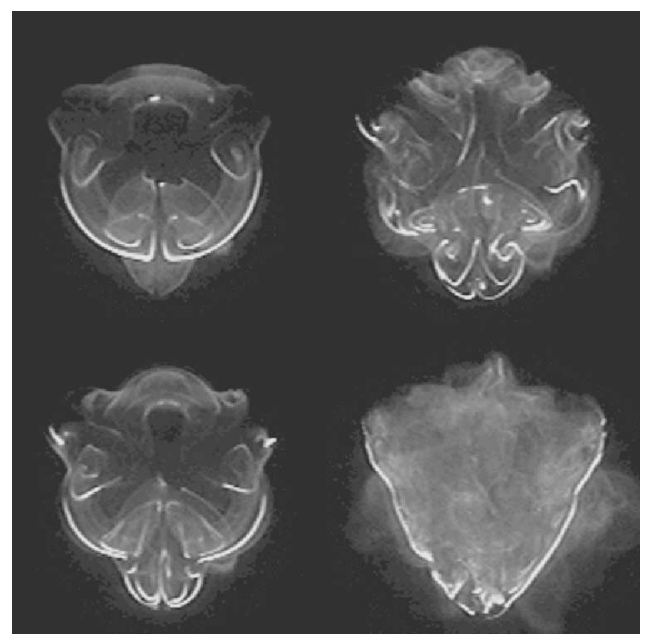

Fig. 4 End views of the starting wake behind a triangular plate

wakes behind an octagonal plate, a hexagonal plate, a square plate and a triangular plate were experimentally compared with that behind a circular disk (Ref. (16)). The starting vortex was deformed due to the stretching of vortices that resulted in longitudinal component of the vorticity. Figure 4 shows the evolution of the wake vortices behind a triangular plate. The experiments were conducted in water to study the initial wake formations behind various polygonal plates placed normal to the starting flow. 
These figures show the end views of the wake development behind a triangular plate. The immediate wake was marked by fluorescent dye and illuminated by a laser sheet parallel to the plate. Three arched (hairpin) vortices are seen crossing the plane of the laser sheet shortly after the flow start-up, and the self-induced velocity causes the inward displacement of the hairpin vortices. The secondary hairpin vortex forms at each of three corners and interact with the primary vortices. A triangular contour of a shear layer is seen in the last image, though a high level of convoluted wake structure exists further downstream even at the steady state.

\subsection{Surface modification}

Effect of surface protrusions on the boundary layer and its separation was demonstrated in a practical situation. Figure 5 (a) and (b) show two velocity vector fields measured using particle image velocimetry on an actual baseball placed in the wind tunnel. The baseball is placed in a so-called 4-seam position. The seam is located upstream in Fig. 5 (a) where the boundary layer is modified and the separation is delayed. In Fig. 5 (b), the boundary layer has already separated and the seam in the reverse flow region has no effect. Since the seam pattern is laid out three-dimensionally, this gives asymmetric flow separation and a large side force that is very sensitive to minute change in the seam orientation. The knuckle ball is effective within a narrow speed range and the ball must be thrown with little spin. Thus the seam and its orientation is more critical than that in cricket ball or roughness in golf balls. This represents the flow physics behind the knuckleball which had been hitherto conjectured (Ref. (17)). On two-dimensional geometry, Amitay et al. ${ }^{(18)}$ used a synthetic jet to modify the pressure distribution on the circu-

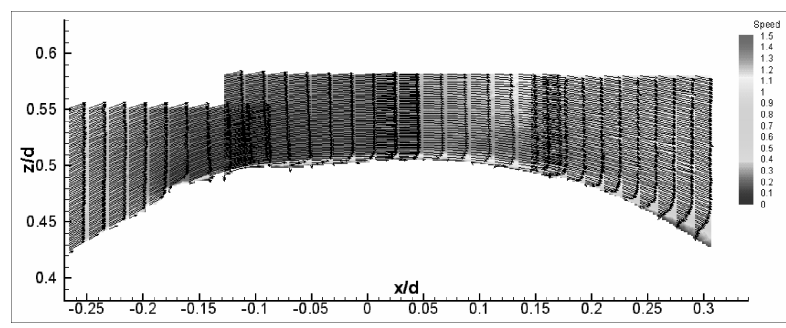

(a) 45 degrees

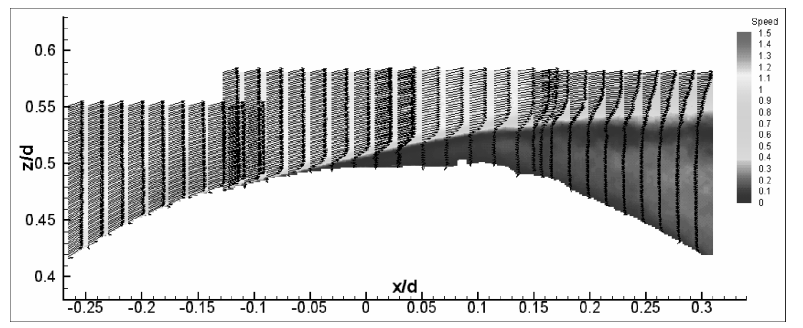

(b) 90 degrees

Fig. 5 Velocity vector field immediately above the surface of a baseball at different seam positions $\left(R e=1 \times 10^{5}\right)$ lar cylinder at lower Reynolds number, but believed to be under slightly different mechanism than the present case.

\section{Active Control}

The experiment on the active control of the disk wake was conducted in the low speed closed-return wind tunnel with a $0.61 \mathrm{~m} \times 0.61 \mathrm{~m}$ test section at Syracuse University. The disk was $10 \mathrm{~cm}$ in diameter and $1.5 \mathrm{~cm}$ in thickness with a beveled edge with downstream face of $9.2 \mathrm{~cm}$ diameter. Six electro-magnetic actuators translated 6 equally spaced segments of the disk edge in radial directions at specified frequency and phase. The stroke of the actuator was limited to $2 \mathrm{~mm}$, and in the retracted position the moveable segments were flush with the remainder of the disk edge ${ }^{(19)}$.

Tabs were actuated at various frequencies but nominally at $\mathrm{x} 1, \mathrm{x} 2, \mathrm{x} 3$ of the frequency corresponding to $m=1$ fundamental frequency (e.g., $15 \mathrm{~Hz}, 30 \mathrm{~Hz}$ and $45 \mathrm{~Hz}$ at $10 \mathrm{~m} / \mathrm{s}$ ). Various modes of actuation tested including the helical excitation. Most of the experiments, however, were conducted with radially symmetric mode or helical mode, as well as the stationary mode as a control. The results of No Actuation, Axisymmetric Actuation ( $m=0$ mode) and Helical Actuation are compared. In the latter neighboring actuators moving in sequence in clockwise (-) or counterclockwise $(+)$ direction viewed from downstream $(m=1$ mode). As shown below no clear distinction in results was seen between the - and + cases during the test.

The time-averaged base pressure was measured on the rear wall at the center of the disk under various tab actuations. When the base pressure was normalized with the natural wake value (pressure coefficient, $C p=-0.49$ ), its dependency on the excitation frequency is clearly shown in Fig. 6. The helical mode of actuation is the most effective at a frequency corresponding to the natural shedding frequency. The direction of helical actuation did not change the outcome. Berger et al. ${ }^{(1)}$ observed that the wake was most responsive to the frequency slightly higher than that of the natural frequency, but at present the change was not discernible within the present frequency sweep increment shown. For the axisymmetric excitation, the change in pressure occurs at twice the frequency of the natural wake, but it is less effective than the helical excitation. In other modes of excitation, the change was almost below the uncertainty of the experiment. Note that the observed decreased base pressure corresponds to the increased drag coefficient as will be further discussed.

The instantaneous velocity vector fields ${ }^{(12)}$ were ensemble-averaged, and the mean velocity fields are shown in Fig. 7 (a) in case of the symmetrical excitation at double the fundamental frequency. The flowfield near the rear stagnation region representing a saddle point was clearly depicted. Zero velocity lines in Fig. 7 (b) indicate that the rear stagnation point of the natural wake is shifted 


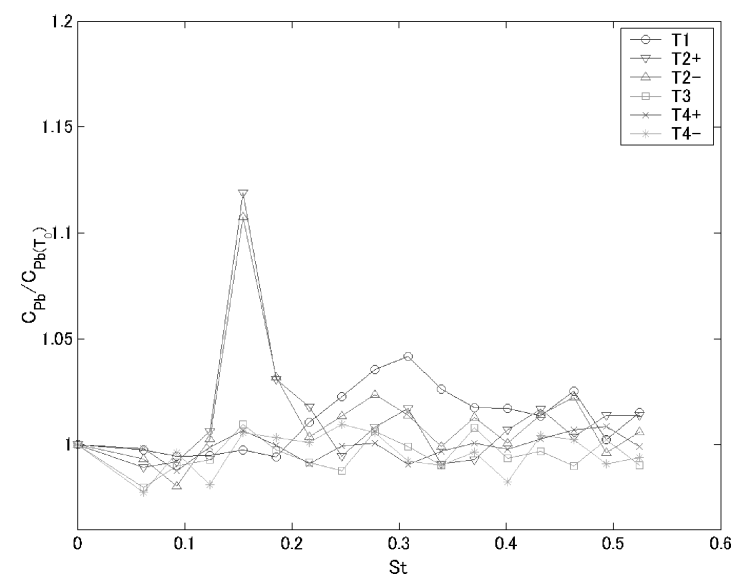

Fig. 6 Base pressure at various actuator excitations (Excitations T1: axisymmetric, T2: helical, T3: 3 tabs 60 degs. apart actuated simultaneously, T4: 2 tabs 180 degs. apart actuated in helical mode)

furthest upstream by the helical excitation at $30 \mathrm{~Hz}$. It is to be noted that the wake width or shear layer curvature is unaffected up to 1 diameter downstream of the wake. The axisymmetric excitation at the double frequency was effectively generating subharmonic helical forcing of the wake. This is similar to the uniform acoustic waves that excited the helical structure in Kim and Durbin's experiment on a sphere ${ }^{(20)}$. The axial velocity profile along the axis depicts the reverse flow region in Fig. 7 (c).

The space-time cross-correlations between the two opposite radial positions were surveyed with hot-wire probes. Anti-symmetric wake oscillations were observed at helical excitation at $15 \mathrm{~Hz}$, but the correlation diminished at higher frequencies. With the axisymmetric excitation at $30 \mathrm{~Hz}$, the anti-phase cross-correlation was observed. In order to examine the time relationship between the excitation and the velocity fluctuation in the shear layer of the wake, the actuator motion and the hot-wire signal in the wake were cross-correlated. With the symmetric excitations the high correlation areas corresponded to the tab actuation frequency and were limited in the radial region directly downstream of the tabs. The helical excitation at the fundamental frequency showed a strong correlation region across the wake, indicating enhancement of large-scale helical vortex structures. Note that these local lock-in frequencies were overshadowed by the fundamental frequency in the overall power spectra. Siegel ${ }^{(21)}$ applied controlled suction and injection at the blunt tail of the sting model in water and surveyed the wake response in detail. When the forcing frequency was scanned, the results at Reynolds number 1500 indicated the maximum spectral peak at the natural frequency as well as lock-in frequency to the forcing. Neither the stereo PIV system or the time-resolved PIV system was capable of measuring the accurate wake oscillation in the velocity vector forms and the hot wire probes were used

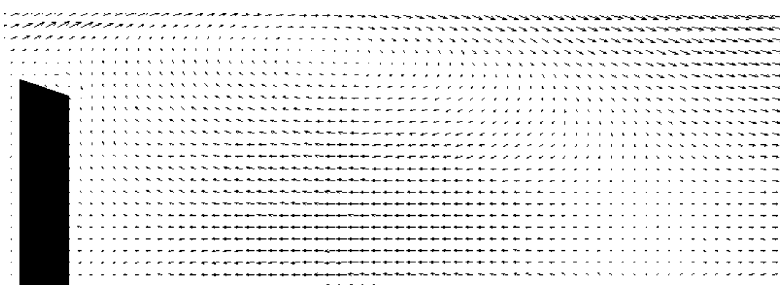

(a) Mean velocity vector: Symmetric excitation $30 \mathrm{~Hz}$

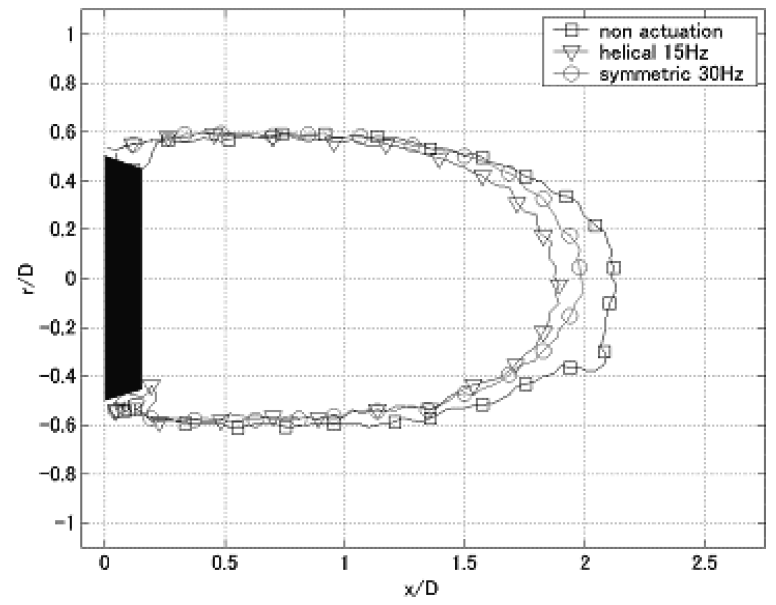

(b) Comparison of zero velocity $(u=0)$ line

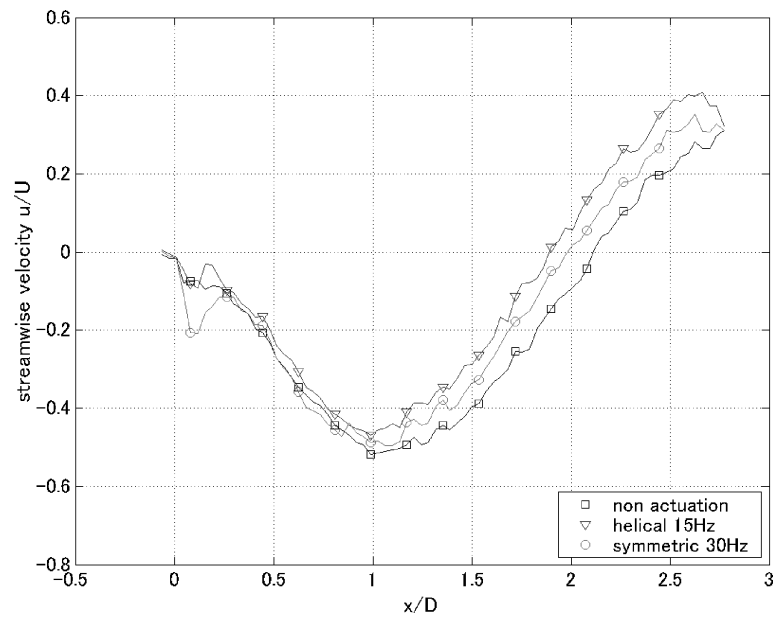

(c) Streamwise velocity along the centerline

Fig. 7 Mean velocity profiles behind the actuated disk

for the time-series analysis. Thus a similar actuated disk experiment was conducted also in water.

The disk model used in the water channel test had 6 slots along the edge and the external pump produced zero-net mass flux actuations. The dimensions of the water channel test section were the same as those of the wind tunnel by design. The results with various actuations agreed in general with those in the wind tunnel, with the additional benefit of applying the time-resolved PIV system. One could interrogate the time-dependent velocity vector field to access the space-time correlation. The study will be continued to access the full capability as well as ascertain the limitations of the system for future imple- 
mentation for feedback systems.

The present tab excitation resulted in decreased base pressure (Fig. 6), i.e., increased drag. As a comparison with the passive control, the tabs were kept in a stationary mode but they did not alter the wake. When stationary flaps as long as $15 \%$ of the disk diameter were fixed at the edge, the wake turbulence was clearly modified generating sufficiently strong longitudinal vortices to influence the wake. Thus it was the active tab movements that enhanced the shear layer vortices and shifted the rear stagnation point upstream and reduced the recirculation bubble in the immediate wake.

\section{Toward Feedback Control}

It is evident that more precise feedback control of the wake structure would be desirable. For a 2-dimensional wake in a low speed water channel, Siegel et al. ${ }^{(22)}$ have successfully implemented a proportional feedback control. The wake behind a disk, however, poses an additional challenge due to multi-mode three-dimensional wake movement. In order to decrease the drag and suppress the helical mode vortices, an alternative flow sensing and control will be required. An active separation control over a wing is concurrently conducted at Syracuse University. The simultaneous PIV velocity field and the multiple pressure fluctuation measurements are used to ascertain and implement the low-dimensional model of flow control ${ }^{(23),(24)}$. A real-time control of the airfoil flow separation using instantaneous surface pressures has been demonstrated. The similar methodology is expected to help in constructing the control strategy of the present disk wake.

\section{Acknowledgement}

The author would like to acknowledge contributions by many who are listed as coauthors in the references and whose collaborations were cited in the present study.

\section{References}

( 1 ) Berger, E., Scholz, D. and Schumm, M., Coherent Vortex Structures in the Wake of a Sphere and a Circular Disk at Rest and under Forced Vibrations, Journal of Fluids and Structures, Vol.4 (1990), pp.231-257.

( 2 ) Higuchi, H. and Balligand, H., Development of Vortex Structure behind a Circular Disk, IUTAM Symposium on Bluff-Body Wakes, Dynamics and Instabilities, Edited by Eckelmann, H., Graham, J.M.R., Huerre, P. and Monkewitz, P.A., (1993), pp.55-58, Springer-Verlag.

(3) Higuchi, H. and Balligand, H., Circulation TimeHistory and Onset of Three-Dimensional Vortex Shedding in the Wake behind a Disk, Proceedings, Second International Symposium on Turbulence and Shear Flow Phenomena, Stockholm, Vol.1 (2001), pp.301306.
( 4 ) Huerre, P. and Monkewitz, P.A., Local and Global Instabilities in Spatially Developing Flows, Annual. Rev. Fluid Mech., Vol.22 (1990), pp.473-537.

( 5 ) Bevilaqua, P.M. and Lykoudis, P.S., Turbulence Memory in Self-Preserving Wakes, J. Fluid Mech., Vol.89 (1978), pp.589-606.

( 6 ) Cannon, S.C., Large-Scale Structures and the Spatial Evolution of Wakes behind Axisymmetric Bluff Bodies, Ph.D. Dissertation, University of Arizona, (1991).

( 7 ) Peterson, C.W., Strickland, J.H. and Higuchi, H., The Fluid Dynamics of Parachute Inflation, Annual Review of Fluid Mechanics, Vol.28 (1996), pp.361-387.

( 8 ) Higuchi, H., Balligand, H. and Strickland, J.H., Numerical and Experimental Investigations of the Flow over a Disk Undergoing Unsteady Motion, J. of Fluids and Structures, Vol.10 (1996), pp.705-719.

( 9 ) Jeon, S., Choi, J., Jeon, W.-P., Choi, H. and Park, J., Active Control of Flow over a Sphere for Drag Reduction at a Subcritical Reynolds Number, J. Fluid Mech., Vol.517 (2004), pp.113-129.

(10) Higuchi, H., Zhang, J., Furuya, S. and Muzas, B.K., Immediate and Near Wake Flow Patterns behind Slotted Disks, AIAA Journal, Vol.36, No.9 (1998), pp.1626-1634.

(11) Naudascher, E., Flow in the Wake of Self-Propelled Bodies and Related Sources of Turbulence, J. Fluid Mechanics, Vol.22, Part 4 (1965), pp.625-656.

(12) Higuchi, H. and Kubota, T., Axisymmetric Wakes behind a Slender Body including the Zero-Momentum Configurations, Physics of Fluids, Vol.2, No.9 (1990), pp.1615-1623.

(13) Sawada, H., Higuchi, H., Kunimasu, T. and Suda, S., Drag Coefficients of Cylinders Magnetically Supported in Axial Flow, Journal of Wind Engineering, (in Japanese), Vol.29, No.4 (2004), pp.55-62.

(14) Higuchi, H. and Sawada, H., Flow over a Magnetically Suspended Cylinder in an Axial Free Stream, AIAA 2005-1078, 43rd AIAA Aerospace Sciences Meeting, Reno, NV, (2005).

(15) Nakamura, M., Onodera, O., Hayasaka, S., Akama, T., Kleine, H., Higuchi, H. and Takayama, K., Performance of a Vertical Two-Stage Gas Gun in the Shock Wave Research Center, Proceedings of the 23rd International Shock Wave Symposium, Texas, (2001).

(16) Higuchi, H., Anderson, R.W. and Zhang, J., ThreeDimensional Wake Formations behind Regular Polygonal Plates, AIAA Journal, Vol.34, No.6 (1996), pp.1138-1145.

(17) Kiura, T. and Higuchi, H., Knuckleball Aerodynamics: Passive Alteration of Three-Dimensional WakeStructure Interactions, IUTAM Symposium on Bluff Body Wakes and Vortex-Induced Vibrations, Marseille, France, (1998).

(18) Amitay, M., Honohan, A.M. and Glezer, A., Flow Control on a Two-Dimensional Circular Cylinder, Third International Symposium on Turbulence and Shear Flow Phenomena, Sendai, Japan, (2003).

(19) Higuchi, H., Ide, S., Qiu, J. and Tani, J., Open-Loop Control of the Disk Wake with Electro-Magnetic Actuators, AIAA Paper 2004-2321, the 2nd AIAA Flow 
Control Conference, (2004), (to Appear in AIAA J.).

(20) Kim, H.-J. and Durbin, P.A., Observation of the Frequencies in a Sphere Wake and of Drag Increase by Acoustic Excitation, Physics of Fluids, Vol.31 (1988), pp.3260-3265.

(21) Siegel, S., Experimental Investigation of the Wake behind an Axisymmetric Bluff Body, Ph.D. Thesis, University of Arizona, AZ, (1999).

(22) Siegel, S., Cohen, K. and McLaughlin, T., Feedback Control of a Circular Cylinder Wake in a Water Tunnel Experiment, AIAA Paper 2004-0580, Reno, NV,
(2004).

(23) Glauser, M., Higuchi, H., Ausseur, J., Pinier, J. and Carlson, H., Feedback Control of Separated Flows, AIAA Paper 2004-2521, 2nd AIAA Flow Control Conference, Portland, Oregon, (2004).

(24) Ausseur, J., Pinier, J., Glauser, M. and Higuchi, H., A Low Dimensional Method for Closed-Loop Feedback Control of the Turbulent Flow over a Wing, AFI/TFI2004 4th International Symposium on Advanced Fluid Information, Sendai, (2004). 